\title{
The research of vehicle's ride comfort in the nonlinear suspension
}

\section{system}

\section{Chen Zhengke}

\author{
Automotive Engineering institute, Jiangxi University of Technology, Nanchang 330098, China
}

Key words: Nonlinear suspension system; Vehicle; Ride comfort; System design

\begin{abstract}
Vehicle's suspension is an important component to ensure ride comfort, which is called the soul of chassis design. The performance of suspension directly affects the vehicle's ride comfort and handling stability. The design of the vehicle's suspension system must fulfill the demand of ride comfort and handling stability. Meanwhile, the suspension, the transmission connecting device between the frame (or body) and the axle (or wheels), is to ensure the safety of driving. It is also one of the indicators to measure the quality of modern automobiles. In the article, it establishes a model of the vehicle nonlinear system for suspension with two degrees of freedom. It uses statistic linearization method to translate the random vibration issue in nonlinear system to that in linear system. Besides, a simulation study is conducted and real cars are tested. The result shows that the suspension's nonlinearity can not be overlooked. Only when the modeling of ride comfort takes account of the suspension's nonlinearity, will the vehicle's ride comfort can be predicted and researched more accurately.
\end{abstract}

\section{Introduction}

When the vehicle is driven, the unevenness of the road surface will bring about vibration of the vehicle. When the vibration reaches to some degree, passengers will feel uncomfortable, or the goods loaded on will be damaged. Meanwhile, the dynamic load between wheels and road surface will affect their adhesion effect, fuel consumption and damage to the vehicle as well as the maneuverability and security of the vehicle, thus causing damage to the road surface. Suspension performance will not only cause the change of car body's posture (pitching and heeling), but also make the passengers uncomfortable and even affect the safety of driving. To improve the vehicle's ride comfort and reduce its vibration, for one thing, the quality of road surface should be improved to reduce vibration. For another, the vehicle should have good vibration isolation. There are three steps for vehicles to reduce vibration, namely, tires, suspension and seats. What plays an important role is the suspension system composed of elastic components and damping elements.

The suspension is the term for all the transmission connecting devices between the frame and axle, which is one of the important assemblies of modern vehicles. The principal function of the suspension is to transmit the wheel's vertical reaction (supporting force), longitude reaction (traction and braking force), side direction reaction and the moment caused by those reactions to the frame, in order to ease the impulse load that the uneven road surface delivers to the vehicle body and to reduce the vibration caused by the impulse load, thus ensuring that the vehicle can be driven normally.

The vehicle's suspension is generally made up of elastic components, absorber and guide mechanism to buffer impact, reduce vibration and transmit force respectively. Elastic components 
can buffer impact and connect the frame (or body) and the axle (or wheels) elastically. In terms of lorries, elastic components are mostly steel plate springs. Besides buffering impact, they are installed in the vehicle vertically and when the articulated juncture joints one end and the frame, they can transmit each force and moment and decide the moving trace of the wheels. Therefore, the guide mechanism can be spared. In addition, average plate springs are folded multiply, so they can reduce vibration to some degree. The vehicle requiring less about vibration reduction can avoid installing absorbers. As for cars, elastic components refer to spiral springs which only bear vertical load to ease and restrain the impact that uneven road surface has on the vehicle's body. They use less space and need no lubrication. However, they themselves have no friction and cannot reduce vibration. Absorber can reduce vibration rapidly (the amplitude decreases quickly). Guide mechanism refers to the cross-style rigid frame like up-down swing arm on the frame and transmission components like knuckle, utilized to transmit vertical force, side force and moment. They can ensure that there is a definite rule of relative motion between wheels and the frame. Those transmission components can also function as guide.

Vehicle's suspension is an important component to ensure ride comfort, which is called the soul of chassis design. The performance of suspension directly affects the vehicle's ride comfort and handling stability. The design of the vehicle's suspension system must fulfill the demand of ride comfort and handling stability. Meanwhile, the suspension, the transmission connecting device between the frame (or body) and the axle (or wheels), is to ensure the safety of driving. It is also one of the indicators to measure the quality of modern automobiles.

It was Booton and Eaughey who put forward statistic linearization method. When O'Caughey applied it to multi-degree system, he adopted regular modal decomposition technique, but it was only fit for the system's mode of vibration and damping matrix. Later, based on the concept that linear components can replace the nonlinear components in the system, Iwan Yang established the united expression of the equivalent parameters of nonlinear components. The physical concept is clear, which avoids solving nonlinear algebraic equations. Afterwards, Atalic and Utku made full use of incentive process featured as a stable Gaussian process and established a more complete and succinct equivalent linear parameter math equation. W.D Wan promoted statistic linear method to multi-degree system and unsteady response and inspected the condition where the solution produced by statistic linearization exists and is unique. It provided large and complex nonlinear response calculation with proper approximate calculation methods.

\section{The development of vehicle's suspension}

At present, most cars adopt traditional passive suspension. The model of its two degree freedom system is shown as Fig.1. The rigidity and damping parameter of this suspension system are selected through empirical design or optimization method. However, since the ride comfort and handling stability contradict the suspension system, a compromise must be made to fulfill the requirement of both performances. Once selected, when the car is driven, the parameters of its suspension characteristic cannot be adjusted to the vehicle's operational states and incentive changes. Hence, the further improvement of its vibration reduction performance is restrained. 


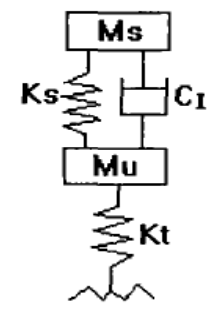

Figure. 1 Passive suspension

As the vehicle's speed is raised, people require more about the ride comfort. To overcome the shortcomings of passive suspension, a new suspension system is sought for to provide better performance. In 1954, Erspiel-Labrosse from GM initially brought forward the idea of active suspension. Active or passive controllable components are adopted to compose a closed-loop control system in the suspension system. It can respond to the vehicle's state of motion and the condition of road surface so as to restrain the motion of the body to leave the suspension constantly in a state of optimal vibration reduction.

In terms of its different working manners, active suspension can be divided into fully active (Fig .1-2), slow active (Fig .1-3) and semi-active (Fig .1-4). Semi-active suspension includes damping level adjustable (O--nOf)f and damping continuously adjustable (Continuous).

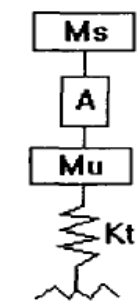

Figure. 2 Full active suspension

\section{The mathematic model of vehicle's suspension system}

When studying a system, we should have an explicit understanding of its physical property as well as a specific expression of the relationship among the internal variables in the system, which means that a systematic mathematic model needs to be established. The specific relationship among the research variables is generated from the fundamental physical laws of the system. Differential equations are utilized for a definitive description.

The vehicle is a complex multi-degree of freedom vibration system. The key to quantitative analysis and ride comfort assessment is to establish a mathematical model to analyze the ride comfort. With the development of computer technology, a multi-degree freedom mathematical model is quite likely to be established, which provides an effective path for the study of vehicle's ride comfort. The research of vehicle's suspension system centres on the system consisting of road, vehicle and passenger. Thus, in order to analyze the relationship between the suspension and ride comfort scientifically, before studying the ride comfort, a vibration mathematic model of the suspension, road model and response assessment criteria should be established.

The vehicle's ride comfort can be analyzed by means of the vibration system diagram. The systematic input is mainly caused by the unevenness which is generated when the vehicle is driven 
on random road surface at a certain speed. It transmits from the vibration system which is composed of the suspended mass of elastic and damping components such as tires, frame and seats and non-suspended mass to suspended mass--- the vehicle's body and human body, both of whose accelerated speeds make up the output in the vehicle's vibration system.

The car is a synthetic moving object. The vibration of its body is caused uneven road surface as well as its components themselves. Meanwhile, the natural vibration frequency of its body, frame and other important components will also have a severe effect on its ride comfort. Hence, every vibration source should be taken into full account in the analysis of the vehicle's ride comfort.

When studying the ride comfort, we must acknowledge human body's vibration characteristics and its physiological reaction. From the experiment, the resonant frequency of human body's up-down vibration approximates $4-6 \mathrm{~Hz}$ while that of front-back vibration is about $\mathrm{ZHz} 25-26$ '. If vibration increases on the point of resonance, human's anti-vibration ability will decline severely because such a vibration reinforces human body's resonance. Therefore, the effect on human body must be considered. Besides up-down vibration, it is found in the experiment that human body is most sensitive to left-right vibration. Front-back vibration is the second and up-down vibration is the most bearable one. Consequently, measures should be taken to reduce the vibration whose frequency is $4-6 \mathrm{HZ}$ when a vehicle is designed.

In general, when constructing the model of ride comfort, we usually take account of 7 degrees of freedom: the body's verticality, pitching, heeling and other 4 of the vertical and horizontal shifts in the centers of mass of front and rear axles. However, some experts believe that the seat's vibration characteristics have a great effect on the ride comfort, for it coordinates human body with the suspension. So, it is necessary to consider the response of the seat and human body to vibration. In addition, with the improvement of road condition and car's lightweight design, the automobile industries inland and overseas attach more and more importance to the effect that the engine's vibration has on the ride comfort, which means it cannot be neglected that the engine's vibration has an impact on the vehicle's performance. The incentive of vibration primarily comes from the road surface and then from the engine and the transmission system as well as the nonuniformity and unbalancedness of the tire's own. Diverse factors along with the dynamic performance of each system constitute the vehicle's reaction.

Suppose there is an engineering system with nonlinear elastic and damping components under a steady and random incentive, linear components are utilized to replace the nonlinear ones. From the viewpoint of statistic linearization, the replacement generates smallest error of mean square. After determining the statistic linear elastic coefficient and damping coefficient, the random vibration issue of the nonlinear system can be transformed to that of linear system.

As with any linear equation, when its equivalent nonlinear equation is used, the coefficient of linear equation set is usually determined by minimizing a certain measurement of the difference of the two sets of equations. So, the solution of linear equation set is the approximate solution of the nonlinear one.

The implementation of statistic linear method can equal a complex nonlinear issue to an easier linear problem. But the rigidity matrix and damping matrix are its specific statistic linear parameter matrix Ke Ce.

Toolbox in the software MATLAB5.3 is utilized to simulate the nonlinear system model and linear system model of the five-degree freedom vehicle' suspension. Since random input is the most essential situation when driving, random input experiment is the most important test to evaluate the 
vehicle's ride comfort. The experiment is conducted by following GB/T4970-96 The random input driving experimental method for vehicle's ride comfort.

Bumping and pit will be encountered when driving. Although it is less likely to happen, too strong impact will affect the ride comfort and even do harm to human's health if it is serious. It is regulated in GB5902-86 The single impulse input driving experimental method for vehicle's ride comfort that triangle single bumping shall be adopted, so the impulse input is a triangle bumping whose bottom length is $400 \mathrm{~mm}$ and height $(\mathrm{h})$ are $60 \mathrm{~mm}, 50 \mathrm{~mm}$ and $120 \mathrm{~mm}$, fit for the total mass that is less than or equal to $4 \mathrm{t}, 4-20 \mathrm{t}$ and greater than $20 \mathrm{t}$.

\section{Conclusion}

The statistic linear principle is applied to deal with the nonlinear problem of the suspension so that the problem can be simplified and not be restrained by the model's degree of freedom. The simulation calculation shows that it is reliable and practical to utilize statistic linear method to solve the random vibration problem in nonlinear system. It is demonstrated in both simulation and real car experiments that there is an obvious difference between the ride comfort prediction of linear suspension and real car experiment. So it is unfit to predict the ride comfort performance. The suspension's nonlinearization factors cannot be neglected in the design and application of suspension. The simulation result and real car experiment show that only if the suspension's nonlinearization factors are taken account of when modeling the ride comfort, will the ride comfort performance be predicted more precisely, thus contributing to guiding the design and improvement of vehicles scientifically.

\section{Acknowledgements}

This work was financially supported by the key subject building project (vehicle engineering) of Jiangxi University of Technology.

\section{References}

[1] Hyldahl P, Andersen S, Mikkelsen S, et al. Modeling Nonlinear Suspension Components in Multibody Systems using nonlinear beam elements based on the Absolute Nodal Coordinate Formulation[R]. SAE Technical Paper, 2015.

[2] Yang Z, Liang S, Sun Y, et al. Vibration suppression of four degree-of-freedom nonlinear vehicle suspension model excited by the consecutive speed humps[J]. Journal of Vibration and Control, 2014: 1077546314543728.

[3] Su X, Yang X, Shi P, et al. Fuzzy control of nonlinear electromagnetic suspension systems[J]. Mechatronics, 2014, 24(4): 328-335.

[4] Safvat R, Mirzaei M, Aghasizade S, et al. Optimal Control of Nonlinear Vehicle Suspension System for Improvement of the ABS Performance[J]. 2014.

[5] Kim B S, Moon B Y, Kim S K. Performance Evaluation of Spring for the Vehicle Suspension System Using the Nonlinear Finite Element Method[C]//Applied Mechanics and Materials. 2014, 635: 594-597. 
[6] Koch G, Kloiber T. Driving state adaptive control of an active vehicle suspension system[J]. Control Systems Technology, IEEE Transactions on, 2014, 22(1): 44-57.

[7] Song S, Duan N, Chen A. Application of variational iteration method for dropping damage evaluation of the suspension spring packaging system[C]//Abstract and Applied Analysis. Hindawi Publishing Corporation, 2014, 2014.

[8] Song S, Duan N, Chen A. Application of variational iteration method for dropping damage evaluation of the suspension spring packaging system[C]//Abstract and Applied Analysis. Hindawi Publishing Corporation, 2014, 2014.

[9] Nam J S, Shin H W, Choi G J. Durability prediction for automobile aluminum front subframe using nonlinear models in virtual test simulations[J]. International Journal of Automotive Technology, 2014, 15(4): 593-601.

[10]Xing B S, Wang N N, Xu L. Study on Nonlinear Damping Properties of Hydro-Pneumatic Suspension System for XP302-Pneumatic Tyred Roller[C]//Advanced Materials Research. 2014, 945: 987-991. 